\title{
Open Knowledge
}

Caycee Neely

Open Knowledge is the principle that innovation and expansion work best when the knowledge about "the how" is available to all. The comprehensive sets of data,

knowledge, technology, and culture focused towards expanding those self-same sets. 